\title{
Development and construction of the Belle II DEPFET pixel detector
}

\author{
Benjamin Schwenker*t \\ Univ. Göttingen \\ E-mail: benjamin.schwenker@phys.uni-goettingen.de
}

\begin{abstract}
The construction of the new accelerator at the Japanese Flavour Factory (KEKB) has been finalized and the commissioning of its detector (Belle II) is planned by early 2017. This new $\mathrm{e}^{+} \mathrm{e}^{-}$ machine ("SuperKEKB") will deliver an instantaneous luminosity of $8 \times 10^{35} \mathrm{~cm}^{-2} \mathrm{~s}^{-1}$, which is 40 times higher than the world record set by KEKB. In order to be able to fully exploit the increased number of events and provide high precision measurements of the decay vertex of the B meson systems in such a harsh environment, the Belle II detector will include a new silicon vertex detector, based on the DEPFET technology. The new pixel detector, located close to the interaction point, consists of two layers of active pixel sensors. The DEPFET technology combines the detection and the in-pixel amplification by the integration, on every pixel, of a field effect transistor into a fully depleted silicon bulk. In Belle II, DEPFET sensors thinned down to $75 \mu \mathrm{m}$ with low power consumption and low intrinsic noise will be used. In this paper, an overview of the latest results and the construction status are presented. Properties of small prototype matrices operated with close to final readout ASIC electronics have been characterized. In particular results from a combined test beam with full-size matrices are described.
\end{abstract}

The 25th International workshop on vertex detectors

September 26-30, 2016

La Biodola, Isola d'Elba, ITALY

\footnotetext{
* Speaker.

${ }^{\dagger}$ On behalf of the DEPFET Collaboration.
} 


\section{Overview of SuperKEKB and the Belle II detector}

The Belle II experiment is a B-factory experiment currently being set up at the site of the High Energy Accelerator Research Organization (KEK) in Tsukuba, Japan, and will take its first physics data in 2018 [1, 2]. It will exploit the unprecedented luminosity $\left(8 \times 10^{35} \mathrm{~cm}^{-2} \mathrm{~s}^{-1}\right)$ of the SuperKEKB accelerator, a fortyfold increase over the predecessor KEKB. The increase in luminosity is achieved on the one hand by roughly doubling the beam currents, on the other hand by a novel nano-beam scheme which leads to a beam size of order $10 \mu \mathrm{m} \times 60 \mathrm{~nm}$ at the interaction point. The implementation of the nano-beam scheme requires a reduced center-of-mass boost compared to the previous B-factory experiments. In order to compensate the thus reduced spatial separation of the B-decay vertices, the new pixel vertex detector (PXD) is placed very close to the interaction point, leading to increased background levels.

Since the PXD is the innermost detector, it will cope with the highest beam-dependent background and is required to keep a high radiation tolerance. Furthermore, in order to reduce multiple scattering, the material budget had to be minimized as much as possible. At the current level of hardware development, the DEPFET technology is perfectly suited to cope with all these requirements [3].

\section{Status of final DEPFET sensor production}

The final production of DEPFET sensors for Belle II is currently running in-house at the MPG HLL semiconductor laboratory. The production consists of 29 SOI wafers and was split into a pilot run ( 3 wafers), a pre-production ( 4 wafers) and a main production (22 wafers). Pilot run and preproduction were successfully finished and delivered several full-size matrices with $250 \times 768$ pixels and test matrices with $32 \times 64$ pixels for test beams and laboratory measurements (see below). The main production was divided into several batches. The fraction of full-size sensors passing all wafer level quality tests at the HLL semiconductor laboratory is roughly $80 \%$ and the main production already now produced enough sensors to equip the Belle II pixel detector. Since the conference, the sensor production was finished.

The basic unit of a DEPFET-based vertex detector (half-ladder) is depicted in figure 1 [4]. A main requirement for the PXD design was to minimize the material budget as much as possible in order to reduce multiple scattering of impinging particles. Concerning this matter, an advantage of DEPFET sensors is that they can be built very thin due to the internal signal amplification and the high signal-to-noise ratio. The sensitive part has a thickness of just $75 \mu \mathrm{m}$. The thinning technology uses anisotropic etching on bonded wafers to create a thin, fully self supporting sensor, where no external structures are needed. The stiffness is provided by a thick $(525 \mu \mathrm{m})$ silicon rim around the sensor, where the auxiliary ASICs are directly bonded to. The thickness of the silicon rim is reduced by etching grooves into it. Furthermore, many parts of the readout electronics are mounted outside of the detector acceptance. There is also no additional supporting material since the PXD is made up of half-ladders of monolithic silicon, which are glued pairwise to form a self supporting structure. 


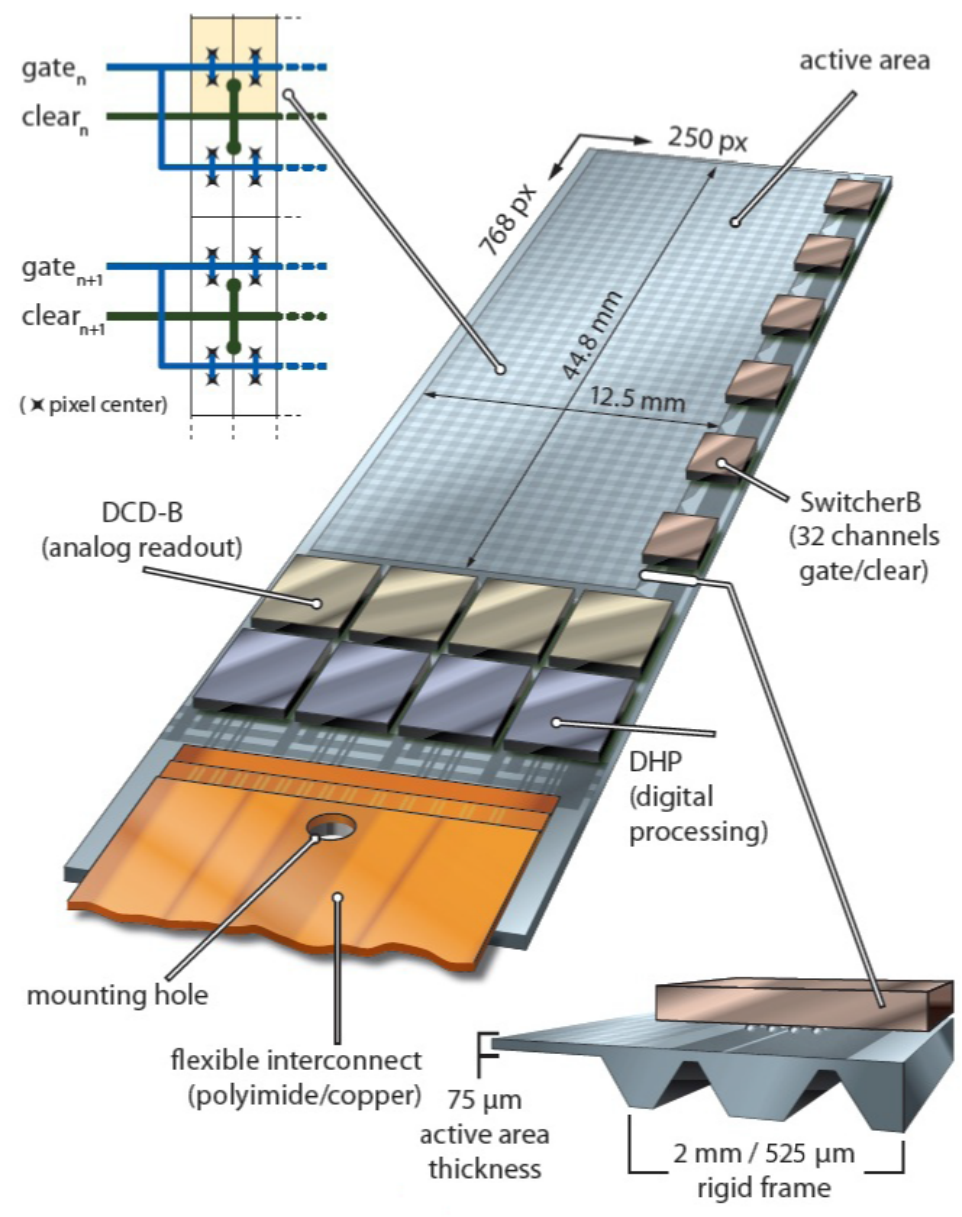

Figure 1: Schematic drawing of the PXD half-ladder.

\section{Performance of readout electronics}

A DEPFET pixel matrix is operated using a rolling-shutter architecture. The gate and clear lines of the pixels, placed on the same matrix row, are connected to a control chip (SwitcherB), which is responsible for selecting subsequent rows. An entire column of pixels is read out by a current receiver ASIC (DCDB or Drain Current Digitizer) [5]. The data from the DCDB is further transmitted to the DHP (Data Handling Processor), the third ASIC on the half-ladder, which performs data processing and compression [6].

A first batch of final readout ASICs for DCDB (version 4.2) and the SwitcherB (version 2.1) and the close to final DHP (version 1.1) became available for detailed tests in May 2016. First successful tests on a probe station were followed by more detailed tests on the small scale detector system shown in figure 2. The test system consisted of a single pair of DCDB and DHP ASICs and allowed measurements of all 256 ADC transfer curves of the DCDB at the design sampling time of 92 ns. Figure 3 shows that the peak-to-peak integral non-linearity (INL) was below 8 ADU for all channels. The median INL was 4.5 ADU. All channels achieve a median noise below 0.6 ADU over the input dynamic range. Two test assemblies were additionally equipped with a SwitcherB 


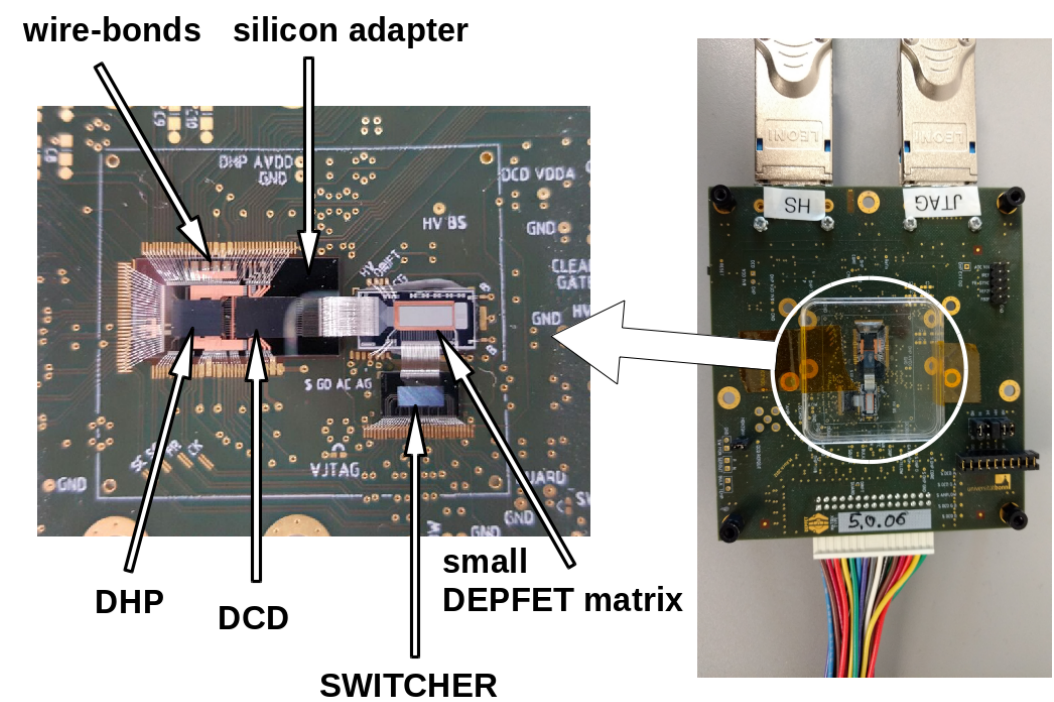

Figure 2: DEPFET test system consisting of a small DEPFET matrix and three readout ASICs assembled on a PCB board. The PCB has two Infiniband connectors for high speed data transmission and slow control.

and a small PXD9 matrix with $32 \times 64$ pixels for source measurements. Figure 4 depicts the cluster charge histogram obtained from a Sr-90 source using a $2 \mathrm{kHz}$ pulse trigger. Although the average noise occupancy was smaller than $10^{-7}$, many events without a Sr-90 signal were accumulated and the peak below the main Landau originates purely from noise hits. The peak of the main Landau is located at $31 \mathrm{ADU}$ and, divided by a single pixel noise of $0.55 \mathrm{ADU}$, leads to a very comfortable signal-to-noise ratio of 55. The in-pixel amplification factor $g_{q}$ was around $700 \mathrm{pA} / \mathrm{e}$ at a drain current of $100 \mu \mathrm{A}$ per pixel. Similar measurements with full-size prototypes are ongoing and results are expected soon.
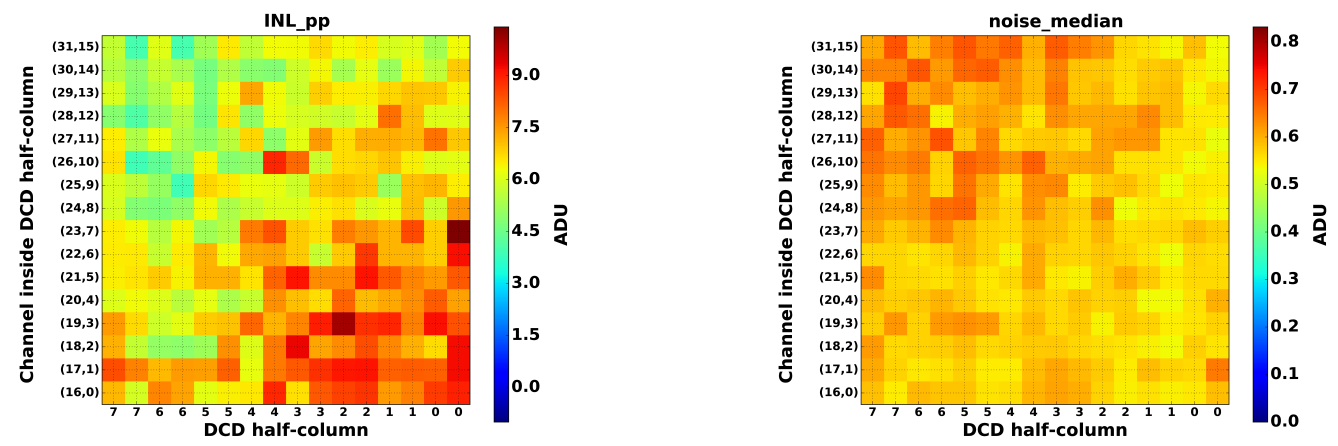

Figure 3: Measurements of analog DCDB (version 4.2) performance at nominal readout speed. The peakto-peak integral non-linearity (left) and median noise (right) are shown for all $16 \times 16$ ADC channels of a DCDB version 4.2 readout ASIC.

\section{Material budget and radiation length imaging}

Material effects like multiple scattering degrade the precision of the reconstructed vertex position and track parameters. In the case of multiple scattering, the magnitude of the effect depends 


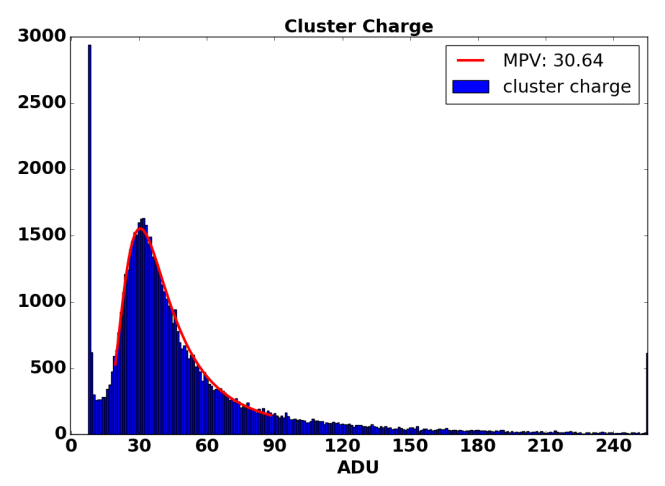

Figure 4: Measurement of a Sr-90 spectrum with a small PXD9 matrix at nominal readout speed.

on the local material budget measured in units of the material constant $X_{0}$. It is therefore crucial to carefully limit and account for every material contribution in the detector acceptance region. The technique of radiation length $X / X_{0}$ imaging is a way to precisely measure the material budget of the PXD half-ladders before installation into Belle II.

At the heart of the $X / X_{0}$ imaging technique is a spatially resolved measurement of the scattering deflection angle $\theta$ of many particles traversing the object under study. To reconstruct the scattering deflection angle and the scattering position, the object under study is placed in the center of a high resolution reference telescope and illuminated by a directed multi $\mathrm{GeV}$ beam of charged particles. The reference telescope provides three upstream and three downstream hits to estimate the projected deflection angles $\theta_{u}, \theta_{v}$ and the intersection coordinates $u, v$ at the object plane. The (local) radiation length $X / X_{0}$ is estimated by fitting the distribution of deflection angles to the Highland formula which is convoluted with a Gaussian density for the angular telescope resolution. More details on radiation length imaging can be found in [7].

In November 2015 a test beam experiment at DESY (Hamburg, Germany) was conducted to acquire the radiation length image for a mechanical prototype of a half-ladder of the Belle II pixel detector. The half-ladder was placed in the center of the EUDET telescope [8] and was illuminated for several hours with a $4 \mathrm{GeV} / \mathrm{c}$ electron beam. The spacings between the telescope planes were approximately $40 \mathrm{~mm}$. At the measurement plane the uncertainty on the track intersection was $3 \mu \mathrm{m}$ and the (calibrated) angular resolution was $150 \mu \mathrm{rad}$. The software for the reconstruction of the beam test data can be found at [9]. Figure 5 shows the measured image (left panel) and compares it to a Geant4 [10] simulation (right panel) of the half-ladder. The simulation involved shooting Geantinos through the Geant4 model of the PXD half-ladder implemented in the Belle II software framework basf2 [11]. Both images show the same area of the half-ladder with a bin side length of $50 \mu \mathrm{m}$. The images show the sensitive area (a) on the left, the $525 \mu \mathrm{m}$ thick silicon balcony with etched grooves covered by a $5 \mu \mathrm{m}$ Cu layer (b) and a SwitcherB (c) as well as air on the right hand side of the images (e). The test beam image even resolves the array of solder bumps (d) which is currently not modeled in basf2. The measured image (left panel) shows that the size of the SwitcherB on the balcony was slightly enlarged for the final design (right panel). The data shows that silicon thickness modulations in the balcony part of the half-ladders are accurately modeled in software and that the PXD achieved the design goal to limit the material budget in the 
tracking acceptance to $2.1 \% X / X_{0}$ for the whole two layer PXD.

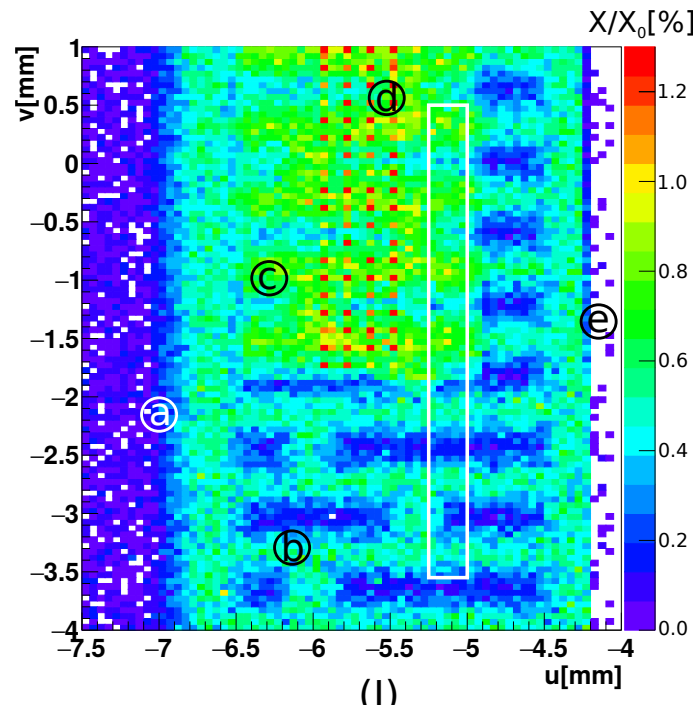

(I)

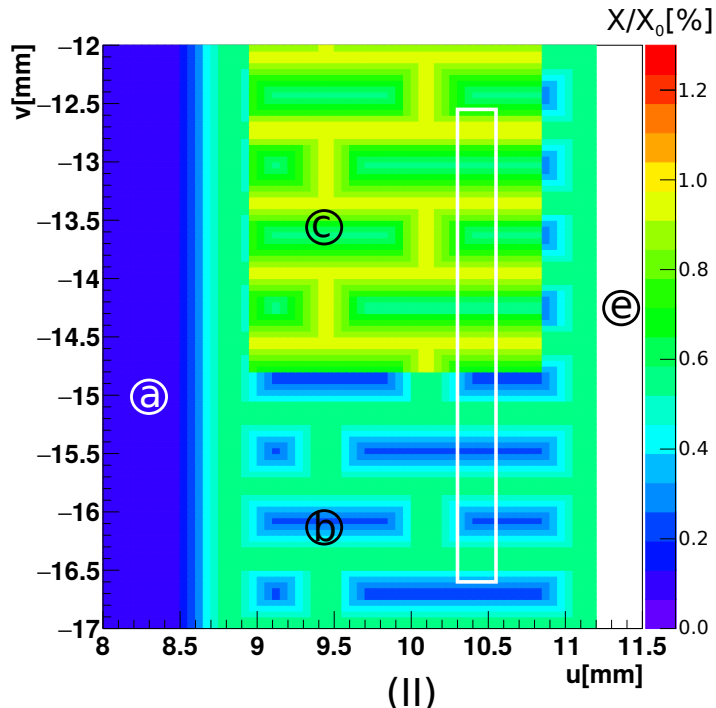

(II)

Figure 5: Beam test data image (I) and simulation image (II). Both images show the same area on a PXD half-ladder. Different parts indicated by the letters (a)-(e) are explained in the main text.

\section{Results from the VXD test beam}

In April 2016, a sector of the Belle II vertex detector was operated in an electron beam at DESY. A schematic drawing of the setup is shown in figure 6. The goal of the test beam period was the combined operation of a small sector of final-design components of the Belle II vertex detector; namely, two final design PXD ladders and four SVD ladders [12], arranged in a configuration that mimics the Belle II vertex detector geometry. The readout of the detector system was performed using a scaled version of the full DAQ chain $[13,14]$. The PXD sensors were biased using the final PXD power supply [15]. The cooling [16] for the VXD used bi-phase $\mathrm{CO}_{2}$, delivered into the detector chamber after $15 \mathrm{~m}$ long distribution lines from the cooling plant. In addition, a full set of environmental monitors (temperature and humidity) based on commercial sensors and calibrated optical fibers with Bragg structures [17] were installed and integrated into the fully developed slow control system.

The test beam setup was inserted in a $1 \mathrm{~T}$ solenoid field. DESY provided a directed electron beam whose momentum was in the $2-6 \mathrm{GeV} / \mathrm{c}$ range with a trigger rate up to $3 \mathrm{kHz}$. A side view illustrating the detector positions and dimensions is shown in figure 7. The beam passed through the coil of a solenoid magnet. Bremsstrahlung processes in the magnet coil lead to a broad momentum distribution of the particles entering the tracker volume. Inside the magnet coil, the beam passed through the upstream arm of the EUDET beam telescope [8] followed by six VXD layers and the downstream arm of the EUDET telescope. The two PXD layers were equipped with two halfladders whose design is shown in figure 1 . The all-silicon half-ladder features a $75 \mu \mathrm{m}$ thin sensor with $250 \times 768$ pixels from the final sensor production for Belle II. As in the final experiment, the long side is parallel to the magnetic field, the bending direction is along the short side. The 
digitization and readout ASICs were chosen from the second to the last generation. Apart from an $20 \%$ increase in the PXD integration time, the front end electronics met all specifications for the Belle II experiment. The inner backward (PXD-IB) and outer backward (PXD-OB) half-ladders were installed on a single support and cooling block. Production delays prohibited the installation of the second cooling block supporting the forward half-ladders.

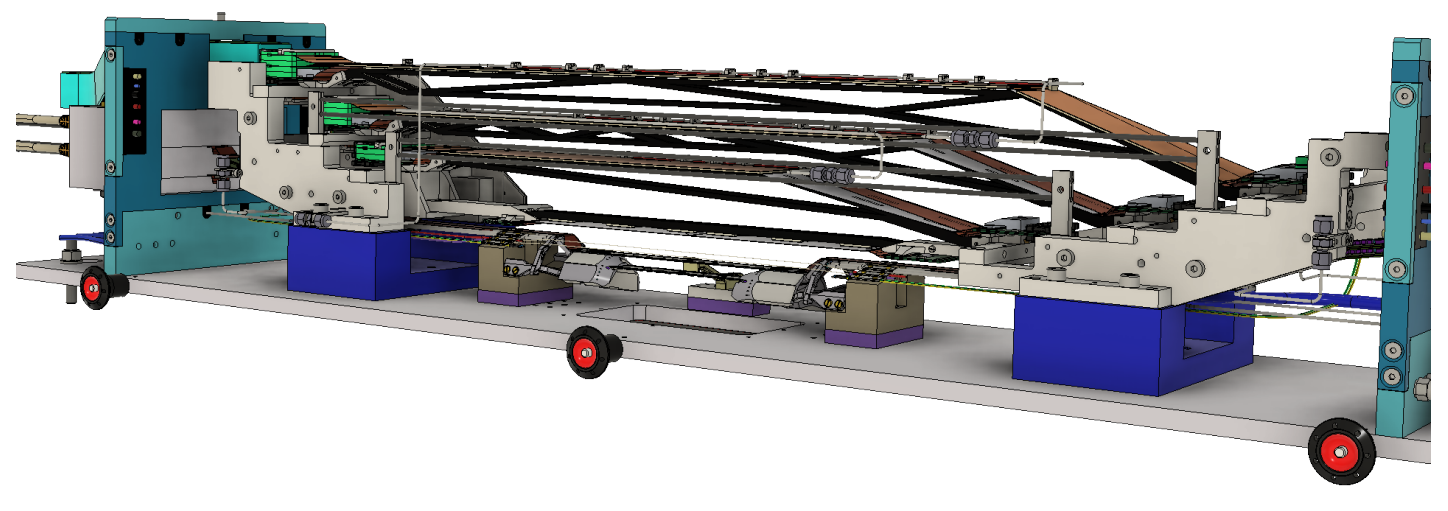

Figure 6: CAD drawing of the VXD setup installed at DESY during the combined test beam. A common ground plate supports the PXD cooling block with two half-ladders and the SVD cartridge with four SVD ladders.

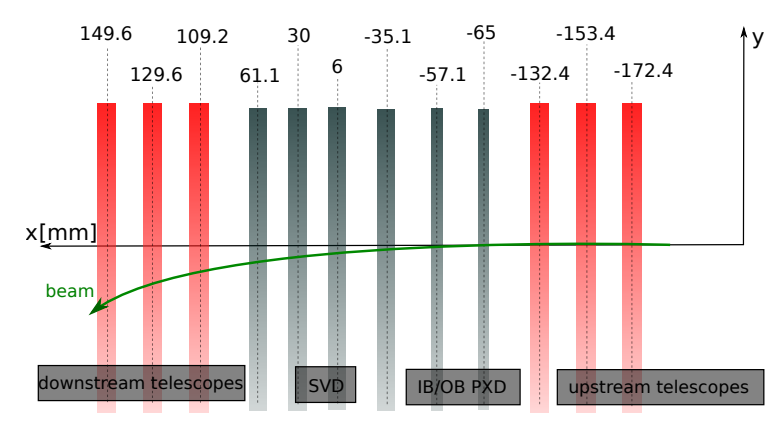

Figure 7: Experimental setup in the test beam experiment. The beam (green) enters from the left where it transverses the coil of the solenoid magnet before reaching the detector assembly consisting of six VXD planes and the EUDET beam telescope.

Downstream of the PXD layers, four SVD layers were installed in a compact cartridge. During the test beam, only the barrel sensors of SVD were illuminated by the electron beam. The barrel sensors in the first SVD layer had an active area of $123 \times 38 \mathrm{~mm}^{2}$ and a thickness of $320 \mu \mathrm{m}$. The double sided barrel sensor had $768 \times 768$ strips, with strip pitches of $160 \mu \mathrm{m}$ and $50 \mu \mathrm{m}$, respectively. It was oriented in the same manner as the PXD layers having the long sensor side parallel to the magnetic field. The barrel sensors in the other three SVD layers had $512 \times 768$ strips 
with a strip pitch of $240 \mu \mathrm{m}$ and $75 \mu \mathrm{m}$, respectively. The full VXD assembly was contained inside a dry volume. The 12 layer tracker consisting of VXD and telescope layers was triggered by the coincidence between two pairs of scintillators placed in front of and behind the assembly. The telescope data was recorded along a separate data path and merged offline based on a common trigger number distributed to the event data.

The test beam setup was used to detect particles, reconstruct their trajectories in real time and to perform online data reduction on the PXD data. The feasibility of this concept was already demonstrated in an earlier VXD test beam campaign at DESY in 2014 [18]. The novelty of the current test beam is the integration of DEPFET sensors from the final sensor production for Belle II. The test beam data offers the possibility to study benchmark variables like the signal distribution, fake rate, hit efficiency and spatial resolution of full-size sensors.
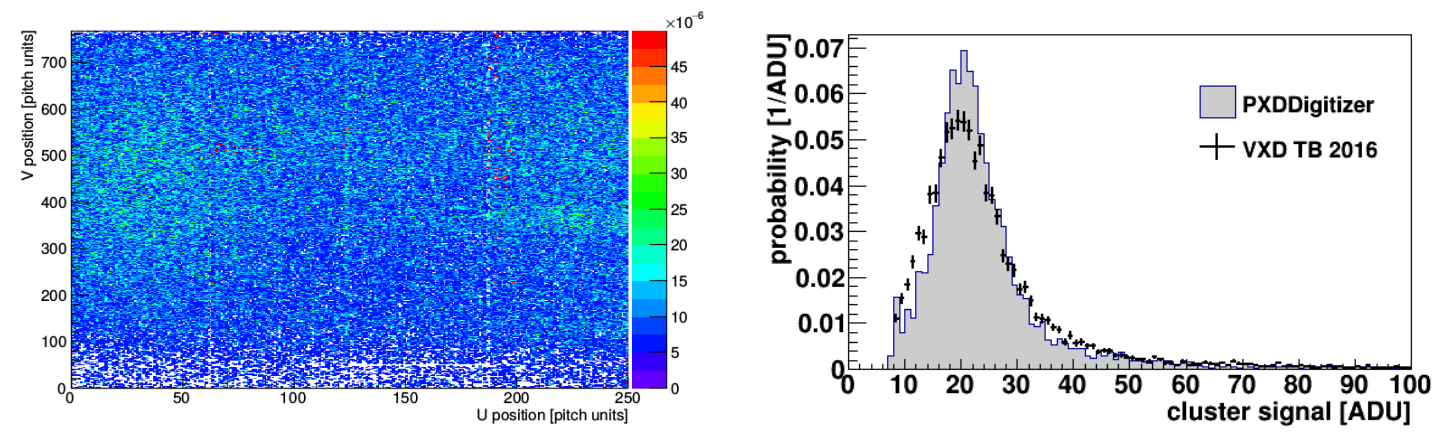

Figure 8: Hit map (left) and cluster charge histogram (right) for the layer 1 PXD sensor during a run in a $1 \mathrm{~T}$ field.

Figure 8 shows the hit map (left) and the cluster charge (right) for the inner layer during a run inside a $1 \mathrm{~T}$ solenoid field. Operation in a solenoid field and wide open collimators delivered a nearly uniform illumination of the $5.6 \mathrm{~mm}^{2}$ large sensitive area. Both PXD half-ladders were found to be fully operational with a fraction of masked pixels well below $0.01 \%$. At a threshold of $7 \mathrm{ADU}$, corresponding to a charge of roughly $1500 \mathrm{e}$, the fake rate per pixel was better than $10^{-6}$ for both sensors. Both PXD sensors were operated in the low gain mode of the DCDB ASIC where the least significant bit had a width of $130 \mathrm{nA}$ and a dynamic range of roughly $30 \mu \mathrm{A}$. The average in-pixel amplification for the whole sensitive area was estimated by comparing histograms for the simulated cluster charge obtained from the PXD detector simulation [19] with the measured cluster charge (right panel, figure 8). The PXD detector simulation takes into account the energy loss straggling, drift and diffusion of charge carriers in the fully depleted silicon sensor, the Lorentz shift of electrons in the magnetic field and the analog-to-digital conversion in the front-end electronics. The histogram obtained in the test beam is broader than predicted because the simulation currently assumes perfectly homogeneous in-pixel gains for all pixels. The average in-pixel amplification is $500 \mathrm{pA} / \mathrm{e}$ at an average drain current of $50 \mu \mathrm{A}$ per pixel and meets the design specifications. The difference to the laboratory measurements on test systems is believed to be caused by the significantly smaller drain currents.

The PXD hit efficiency was studied using a several hours long run with a solenoid field of $1 \mathrm{~T}$ and a $4 \mathrm{GeV} / \mathrm{c}$ electron beam. A time delay prohibited the implementation of the PXD9 specific 
channel mapping in the PXD DAQ. As a consequence, the correct mapping was applied as a step in the offline data processing after the event builder. In order to bypass the missing mapping, a set of runs was selected for the efficiency study where the online data reduction was deactivated and all pixel data was written to disk. The official Belle II software was used to find a sample of tracks having at least three SVD hits. After a track was fitted based on hits in the SVD layers, the definition of the region of interest (ROI) took place. Regions of interest were defined as rectangular areas on the PXD sensors which are likely to have been crossed by the fitted tracks. The ROIs were found by finding the intercepts of a track with the two PXD sensors and then constructing a rectangular area around the intercept. Only events with a single high momentum track $(>1 \mathrm{GeV} / \mathrm{c})$ and a track fit p-value above 0.01 were used to avoid problems with low momentum tracks. The size of the region of interest was chosen to be $0.4 \times 0.4 \mathrm{~mm}^{2}$.
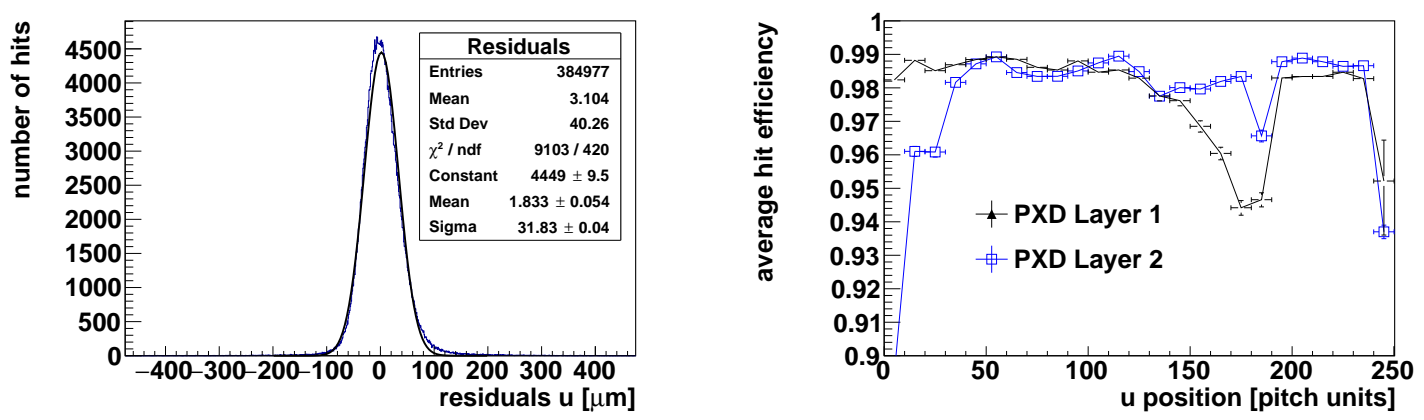

Figure 9: Residuals (left) between SVD tracks and PXD clusters on the inner layer and hit efficiency (right) for both PXD layers.

Figure 9 (left) shows the histogram of residuals between selected tracks and clusters inside the region of interest along the short $(u)$ side of the sensor. The residual width is dominated by the extrapolation uncertainty of the SVD tracks to the PXD sensor in a $1 \mathrm{~T}$ field including low momentum particles down to $1 \mathrm{GeV}$. For the efficiency measurement, a track was counted as a pass when a PXD cluster was found in the region of interest on the same sensor. The probability to create a false pass by finding a fake cluster inside the region of interest was estimated to be smaller than $10^{-3}$. Figure 9 (right) gives the hit efficiency measured in 25 bins corresponding to groups of ten matrix columns. In 18 out of 25 bins the efficiency was above $98 \%$ for the inner layer while the outer layer achieved an efficiency above $98 \%$ in 20 bins. All inefficient bins also showed a strong downward shift in the most probable cluster charge and more detailed investigations are ongoing.

The spatial resolution of the PXD sensors was studied by combining the hits from the six VXD planes with hits from the EUDET telescope. The geometrical acceptance for electron tracks with hits in both the VXD and the telescope was rather small and only one such track was found in 100 events. Unfortunately, the telescope was only running during a few hours of beam time and telescope studies were limited to a few thousand tracks. As an example, figure 10 shows the residuals $u_{\text {hit }}-u_{\text {fit }}$ for the outer PXD sensor in a run without magnetic field and a $5 \mathrm{GeV} / \mathrm{c}$ electron beam. The track intersection coordinate $u_{f i t}$ is estimated using hits from at least three SVD planes and six telescope planes. The estimated uncertainty of the intersection coordinate was $5 \mu \mathrm{m}$ because of the precise hit information from the telescope plane. The fraction of double 
pixel clusters of about $18 \%$ originates from the nearly perpendicular incidence of electrons into the sensor. The residual RMS for single hit clusters agrees well with the digital resolution of Pitch $/ \sqrt{12} \approx 14.3 \mu \mathrm{m}$. On the other hand, the spatial resolution for two pixel clusters is much better due to the charge sharing between hit pixels.
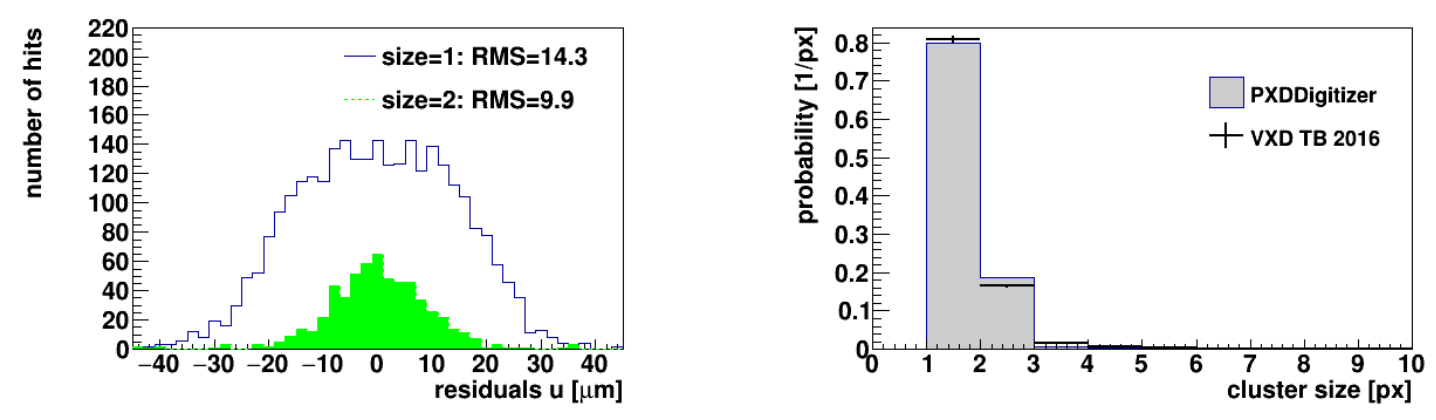

Figure 10: Residuals (left) and cluster size (right) along the short sensor side (50 $\mu \mathrm{m}$ pitch) measured on the outer backward PXD sensor.

\section{Summary}

During the test beam campaign in April 2016, a sector of the Belle II vertex detector consisting of four SVD ladders and two PXD half-ladders was successfully integrated with the Belle II DAQ system. The test beam achieved a common readout of PXD and SVD subsystems. Online, realtime processing of SVD data using the Belle II software framework's network data distribution and parallel processing capabilities was demonstrated and confirmed results from earlier test beam campaigns.

The performance of two full-size PXD sensors was studied for the first time. Both sensors were found to be fully operational with a comfortable signal-to-noise ratio and a low fraction of noisy pixels $(0.01 \%)$. A first calibration of the cluster charge distributions shows reasonable agreement with the detector simulation confirming that in-pixel amplification $g_{q} \approx 500 \mathrm{pA} / \mathrm{e}$ was achieved. The average hit efficiency was found to be better than $98 \%$ for most parts of the sensor area. A study of the spatial resolution demonstrated the improvements from charge interpolation in multi pixel clusters.

Since November 2016, the two PXD sensors from the April test campaign including the complete PXD DAQ chain were successfully installed into the permanent running system at DESY (PERSY). At this stage, PERSY will be used to improve system integration and to test the implementation of missing features like the PXD9 specific mapping. Then PERSY will be extended for the purpose of commissioning the two PXD half-shells before transport to KEK in late 2017.

\section{Acknowledgments}

This work was supported by the German Federal Ministry for Research BMBF. The research leading to these results has received funding from the European Commission under the FP7 Research Infrastructures project AIDA, grant agreement no. 262025. 


\section{References}

[1] T. Abe et al., Belle II Technical Design Report, 2010.

[2] A. Bevan et al., The Physics of the B Factories, Eur. Phys. J., vol. C74, no. 11, p. 3026, 2014.

[3] C. Marinas et al. [DEPFET Collaboration], The Belle II pixel detector: High precision with low material, Nucl. Instrum. Meth. A 731 (2013) 31.

[4] H. G. Moser, L. Andricek, R. H. Richter and G. Liemann, Thinned silicon detectors, PoS VERTEX2007 (2007) 013.

[5] I. Peric, P. Fischer and T. H. H. Nguyen, DCDB and SWITCHERB, the readout ASICS for Belle II DEPFET pixel detector, IEEE Nucl. Sci. Symp. Conf. Rec. 2011 (2011) 1536.

[6] M. Lemarenko, T. Hemperek, H. Krüger, M. Koch, F. Lütticke, C. Marinas and N. Wermes, Test results of the data handling processor for the DEPFET pixel vertex detector, JINST 8 (2013) C01032.

[7] U. Stolzenberg et al., Radiation length imaging with high-resolution telescopes, to be published in Nucl. Instr. Meth. A, arXiv:1609.02402

[8] A. Bulgheroni, Results from the EUDET telescope with high resolution planes, Nucl. Instrum. Meth., vol. A623, 2010.

[9] Test beam software framework, https://bitbucket.org/BenjaminSchwenker/tbsw

[10] J. Allison et al., Geant4 developments and applications, IEEE Transactions on Nuclear Science 53 (2006) 270-278

[11] R. Itoh et al., Implementation of parallel processing in the basf2 framework for Belle II, J. Phys. Conf. Ser., vol. 396, 2012.

[12] M. Friedl et al., The Belle II Silicon Vertex Detector, Nucl. Instrum. Meth., vol. A732, 2013.

[13] D. Münchow, J. Dingfelder, T. Gessler, I. Konorov, W. Kühn, S. Lange, K. Lautenbach and D. Levit et al., The data acquisition system of the Belle II Pixel Detector, JINST 9 (2014) C08009.

[14] S. Lee et al., Development of high level trigger software for Belle II at SuperKEKB, J. Phys. Conf. Ser., vol. 331, p. 022015, 2011.

[15] S. Rummel et al., The power supply system for the DEPFET pixel detector at BELLE II, Nucl. Instrum. Meth. A 699 (2013) 51.

[16] P. Ruiz-Valls et al. [DEPFET Collaboration], The ultra low mass cooling system of the Belle II DEPFET detector, Nucl. Instrum. Meth. A 731 (2013) 280.

[17] D. Moya and I. Vila, Structural and environmental monitoring of tracker and vertex systems using Fiber Optic Sensors, arXiv:1203.0109 [physics.ins-det].

[18] T. Bilka, G. Casarosa, R. Frühwirth, C. Kleinwort, P. Kodys, P. Kvasnicka, J. Lettenbichler and E. Paoloni et al., Demonstrator of the Belle II Online Tracking and Pixel Data Reduction on the High Level Trigger System, arXiv:1406.4955 [physics.ins-det].

[19] Z. Drasal, K. Prothmann, and B. Schwenker, Silicon simulation code for Belle II and ILC, PoS VERTEX 2011 (2011). 